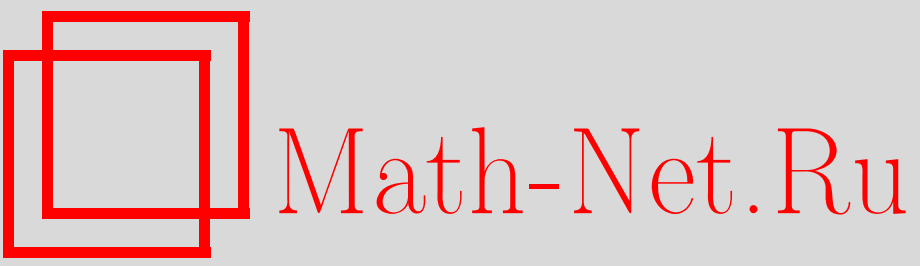

А. В. Васюков, М. А. Еловенкова, И. Б. Петров, Деформирование и разрушение тонкой нити под действием динамической нагрузки, Матем. моделирование, 2020, том 32, номер 5, 95-102

DOI: https://doi.org/10.20948/mm-2020-05-05

Использование Общероссийского математического портала Math-Net.Ru подразумевает, что вы прочитали и согласны с пользовательским соглашением

http://www . mathnet.ru/rus/agreement

Параметры загрузки:

IP: 54.84 .234 .179

26 апреля 2023 г., 11:31:00 


\title{
ДЕФОРМИРОВАНИЕ И РАЗРУШЕНИЕ ТОНКОЙ НИТИ ПОД ДЕЙСТВИЕМ ДИНАМИЧЕСКОЙ НАГРУЗКИ
}

\author{
(C) 2020 2. $\quad$ А.В. Васюков, М.А. Еловенкова, И.Б. Петров
}

Московский физико-технический институт a.vasyukov@phystech.edu

Работа выполнена при поддержке гранта РФФИ № 18-29-17027.

DOI: $10.20948 / \mathrm{mm}-2020-05-05$

Рассматривается задача распространения деформаций и напряжений в тонкой нити при действии механической ударной нагрузки, а также разрушение нити в данном процессе. Рассматриваются высокоскоростные взаимодействия, для которых скорость движения ударника сопоставима со скоростью распространения звука в нити. Для верификации выполнено моделирование развития деформаций в одномерной нити под действием точечного удара, результаты сопоставлены с аналитическим решением. Приведены расчёты различных режимов воздействия распределённой нагрузки на тонкую нить, приводящих к принципиально разной картине деформаций и разрушений нити.

Ключевые слова: математическое моделирование, тонкая нить, ударная нагрузка, разрушение.

\section{MODELING OF THIN FIBER DEFORMATION AND DESTRUCTION UNDER DYNAMIC LOAD}

\section{A.V. Vasyukov, M.A. Elovenkova, I.B. Petrov}

Moscow Institute of Physics and Technology (state university)

The problem of the propagation of deformations and stresses in a thin fiber under the dynamic mechanical load is considered, as well as the destruction of the fiber. High-speed interactions are considered, for which the striker speed is comparable to the sound speed in the fiber. The numerical results are compared with an analytical solution for a point impact. Various loading modes are calculated for a distributed load, that cause significantly different types of deformation and destruction of the fiber.

Key words: numerical simulation, thin fiber, dynamic load, destruction.

\section{Введение}

Задача распространения деформаций и напряжений в тонкой нити при действии динамической нагрузки тесно связана с задачей динамической 
прочности тканевых композитов, сплетённых из индивидуальных нитей. Тканевые композиты на сегодняшний день применяются во многих областях техники, в том числе в защитных экранах космических аппаратов. В данной области применения тканевых композитов характерной внешней нагрузкой, от которой должен обеспечить защиту экран, являются удары микрометеоритов и космического мусора. Данная задача обладает рядом особенностей, основной из которых является скоростной режим взаимодействия - характерная скорость метеорита или обломка может составлять 5-7 км/с. Такой скоростной режим крайне сложно изучать экспериментально, в силу чего исследования методами математического моделирования становятся особенно актуальны.

Задачи динамической прочности тканевых композитов достаточно хорошо изучены для низких скоростей взаимодействия и одиночного массивного ударника. Накоплена обширная экспериментальная база, существует опыт выполнения оценок и построения эмпирических моделей для данных режимов взаимодействия [1]. Также существует опыт прямого расчёта прочности тканевого композита с использованием моделей различных видов, учитывающих более или менее сложные эффекты взаимодействия нитей и слоёв внутри тканевого пакета. Наиболее простой и при этом активно используемой является модель «крест-колокол» [1], в которой считается, что основной вклад в суммарную силу сопротивления ударнику дают нити, проходящие непосредственно через область воздействия ударника на тканевый пакет. При известном радиусе ударника суммарное число нитей, принимающих участие во взаимодействии, несложно определяется из его геометрического размера, поверхностной плотности ткани и плотности материала волокна. При этом в рамках модели нити рассматриваются как невзаимодействующие, каждая нить независимо взаимодействует с ударником, их вклад в его торможение суммируется.

Применение модели «крест-колокол» для быстрых оценок представляется разумным, так как на образцах со сквозным пробитием визуально наблюдается характерный «крест» из вытянутых нитей, на которые пришлась основная нагрузка в ходе взаимодействия с ударником (рис.1).

В работах других авторов встречаются и более сложные модели [2-4] с базовым учётом взаимодействия нитей, приводящим к пирамидальной форме деформированной области, а также модели изотропной мембраны.

Однако все данные модели исходно построены в предположении, что скорость ударника много меньше скорости звука в нитях тканевого экрана. В силу этого прямое обобщение моделей на рассматриваемый скоростной режим было бы некорректным. 
Существуют аналитические решения для взаимодействия ударника с гибкой нитью, полученные в работах Рахматулина с соавторами [5]. Полученные простые аналитические соотношения справедливы для случая удара с постоянной скоростью по нити бесконечной протяженности. При ударе телом конечной массы конфигурация нити в поперечной волне будет криволинейной и вычисление значительно усложняется. Приближенное аналитическое решение задачи об ударе по нелинейной нити телом конечной массы также приведено в указанной работе.

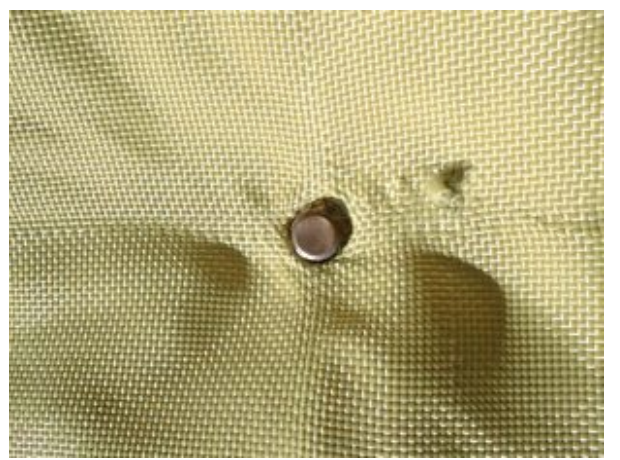

Рис.1. Пример образца ткани со сквозным пробитием, по данным [1]

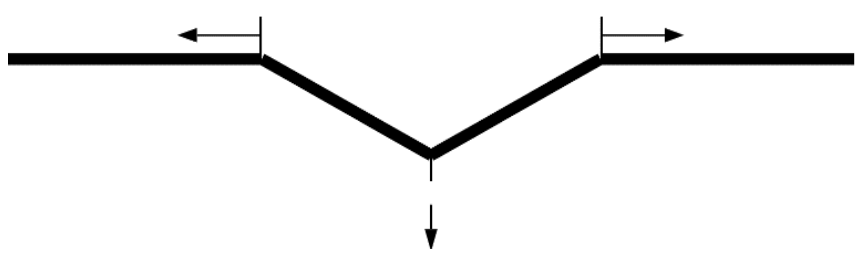

Рис.2. Общий вид деформированной нити при поперечном ударе.

\section{Математическая модель}

В данной работе рассматривается отдельная тонкая гибкая нить. Толщина нити принимается постоянной и не входит в уравнения, описывающие поведение нити. Диаметр нити задаётся как один из параметров расчёта, используется для определения массы сегмента нити и пересчёта внешнего давления в силу воздействия. Нить рассматривается как одномерный объект в трёхмерном пространстве. Для описания нити рассматривается следующая система уравнений $[5,6]$ :

$$
\begin{aligned}
& \rho_{0} \frac{d^{2} x}{d t^{2}}=\frac{\partial(T \cos \varphi)}{\partial s_{0}}+\rho_{0} P \sin \varphi \\
& \rho_{0} \frac{d^{2} y}{d t^{2}}=\frac{\partial(T \sin \varphi)}{\partial s_{0}}+\rho_{0} P \cos \varphi .
\end{aligned}
$$

Здесь $\rho_{0}$ - начальная плотность материала нити, принимаемая постоянной в ходе расчёта, $s_{0}$ - расстояние вдоль нити, $T$ - сила натяжения нити, $\varphi$ - угол между осью $O x$ и вектором касательной к нити, $P$ - внешняя сила, действующая перпендикулярно нити.

Сила натяжения действует вдоль нити и связана с относительным удлинением $\varepsilon$ линейным образом: $T=\varepsilon \cdot E$. 
В расчётах с разрушением обрыв нити в точке происходит при достижении в данной точке критического удлинения $\varepsilon_{k}$.

Для численного решения данной системы уравнений используется явная двухслойная трёхточечная разностная схема, обладающая вторым порядком аппроксимации по пространству и первым - по времени [7].

\section{Верификация}

На рис.2 показан общий вид деформированной нити для случая точечного поперечного удара с постоянной скоростью по нити бесконечной протяженности [6]. Пунктирной стрелкой показана точка и направление удара. Сплошными стрелками показано направление движения точек излома нити вдоль нити.

Для данной постановки аналитическое решение для скорости распространения волны сильного разрыва (скорости движения излома нити вдоль нити) имеет вид:

$$
u=0.8 a_{0}^{1 / 3} v_{0}^{2 / 3},
$$

где $a_{0}=\sqrt{E / \rho}-$ скорость распространения звука в нити, $v_{0}-$ скорость ударника.
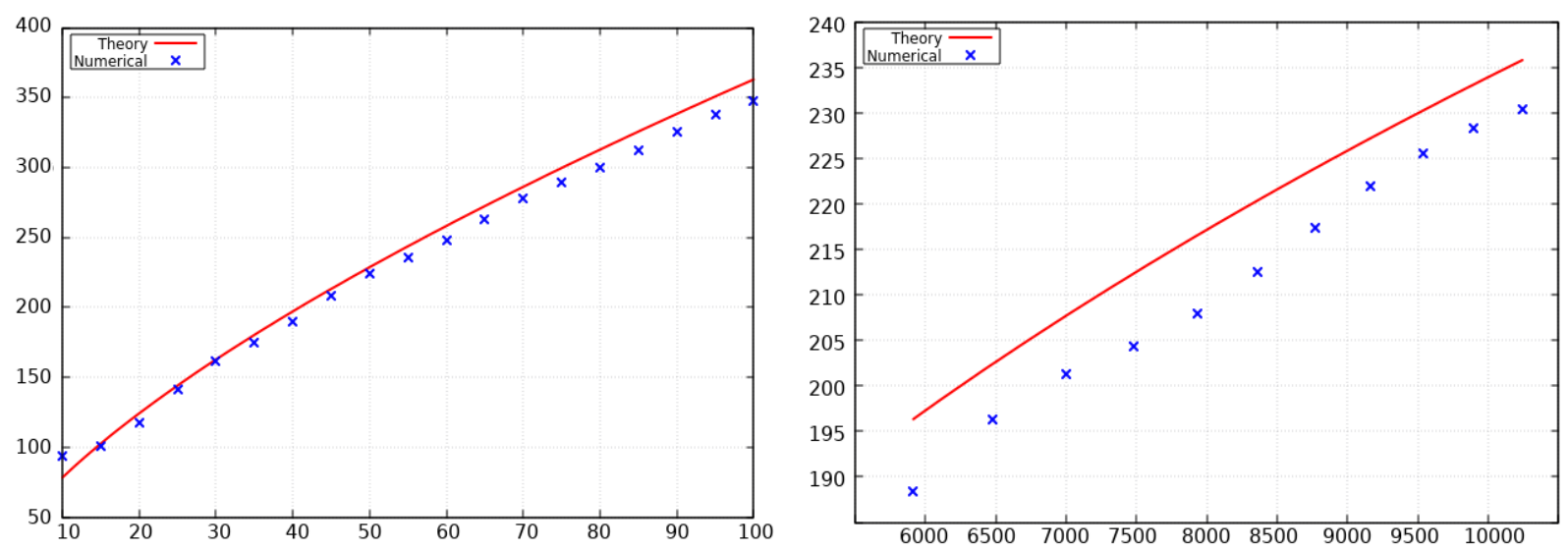

Рис.3. Зависимость скорости излома нити (по оси ОY) от скорости удара (по оси ОХ) для точечного удара по нормали. Модуль Юнга нитей 125 ГПа, плотность материала нитей 1430 кг/м³. Сплошная линия - аналитическое решение, точки - результаты расчётов.
Рис.4. Зависимость скорости излома нити (по оси OY) от скорости звука в нити (по оси OX) для точечного удара по нормали. Модуль Юнга варьируется в диапазоне 50-150 ГПа, плотность материала нитей $1430 \kappa г / \mathrm{M}^{3}$. Скорость удара 50 м/с. Сплошная линия - аналитическое решение, точки - результаты расчётов. 
Для верификации выбранной модели и численной схемы для аналогичной постановки численно исследовалась зависимость скорости движения излома нити от скорости движения ударники и от скорости звука в материале нити. Так как аналитическое решение предполагает бесконечность нити, то для корректности прямого сравнения в численном решении длина нити выбиралась достаточной для того, чтобы за время расчёта возмущения не достигли её концов. Базовые значения параметров расчёта: $L=0.1 \mathrm{м}$ - длина нити, $D=0.0001$ м - диаметр нити, $E=125$ ГПа - модуль упругости нити, $\rho=1430$ кг $/ \mathrm{M}^{3}$ - плотность материала нити. Далее скорость удара варьировалась в диапазоне 10-3000 м/с, модуль упругости - в диапазоне 50-150 ГПа. Полученные зависимости приведены на рис.3, 4. В данных расчётах мелкость сетки составляла 50 точек на длину нити.

\section{Результаты расчетов}

После верификации была выполнена серия расчётов различных режимов с разрушением, результаты приведены на рис.5-8.

Параметры данных расчётов:

- $\quad$ диаметр нити 0.1 мм;

- $\quad$ длина нити $10 \mathrm{cm;}$

- $\quad$ концы нити не закреплены;

- $\quad$ плотность материала нити $1430 \mathrm{\kappa г} / \mathrm{m}^{3}$;

- модуль Юнга материала нити 125 ГПа;

- $\quad$ критическое удлинение материала нити 4\%;

- $\quad$ размер области действия давления $1 \mathrm{cm;}$

- $\quad$ форма импульса давления по пространству $\cos ^{2}(r)$;

- длительность импульса давления 10 мкс;

- $\quad$ амплитуда давления варьировалась от 20 до 40 МПа.

На рис.5-8 видно, что волновые процессы в нити приводят к формированию сложной картины как деформаций, так и разрушений. При изменении амплитуды внешнего воздействия можно получить как разрыв нити посередине, так и аналог «тыльного откола» на концах нити или выбивание элемента нити с последующим его дроблением волнами, «запертыми» в выбитом элементе. 

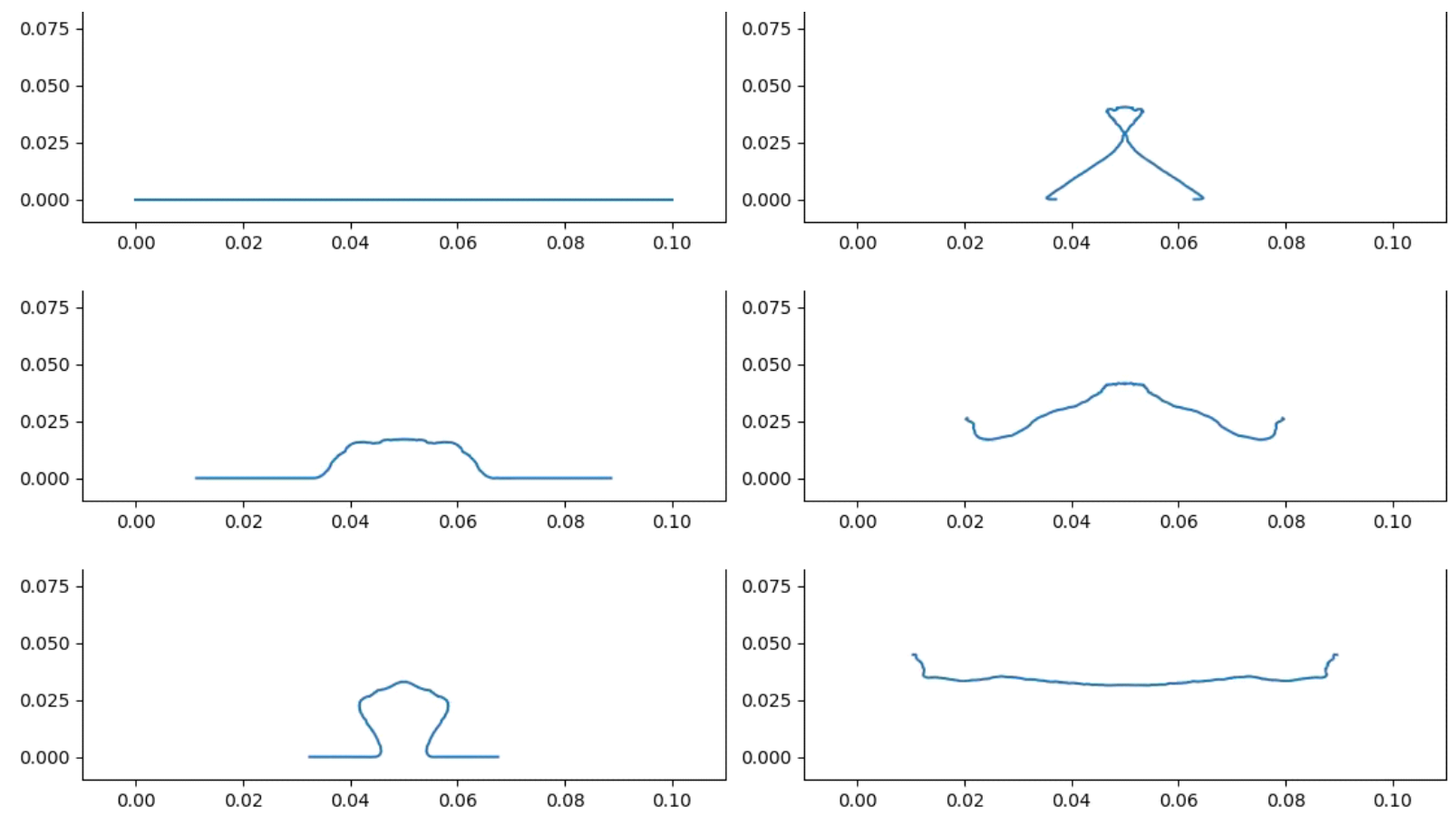

Рис.5. Развитие деформаций в нити. Внешнее давление 25 МПа. Нить остаётся целой. Левый столбик сверху вниз - моменты времени 0, 8, 16 мкс. Правый столбик сверху вниз - моменты времени 24, 32, 40 мкс. Координаты точек нити по осям ОХ и ОY в метрах.
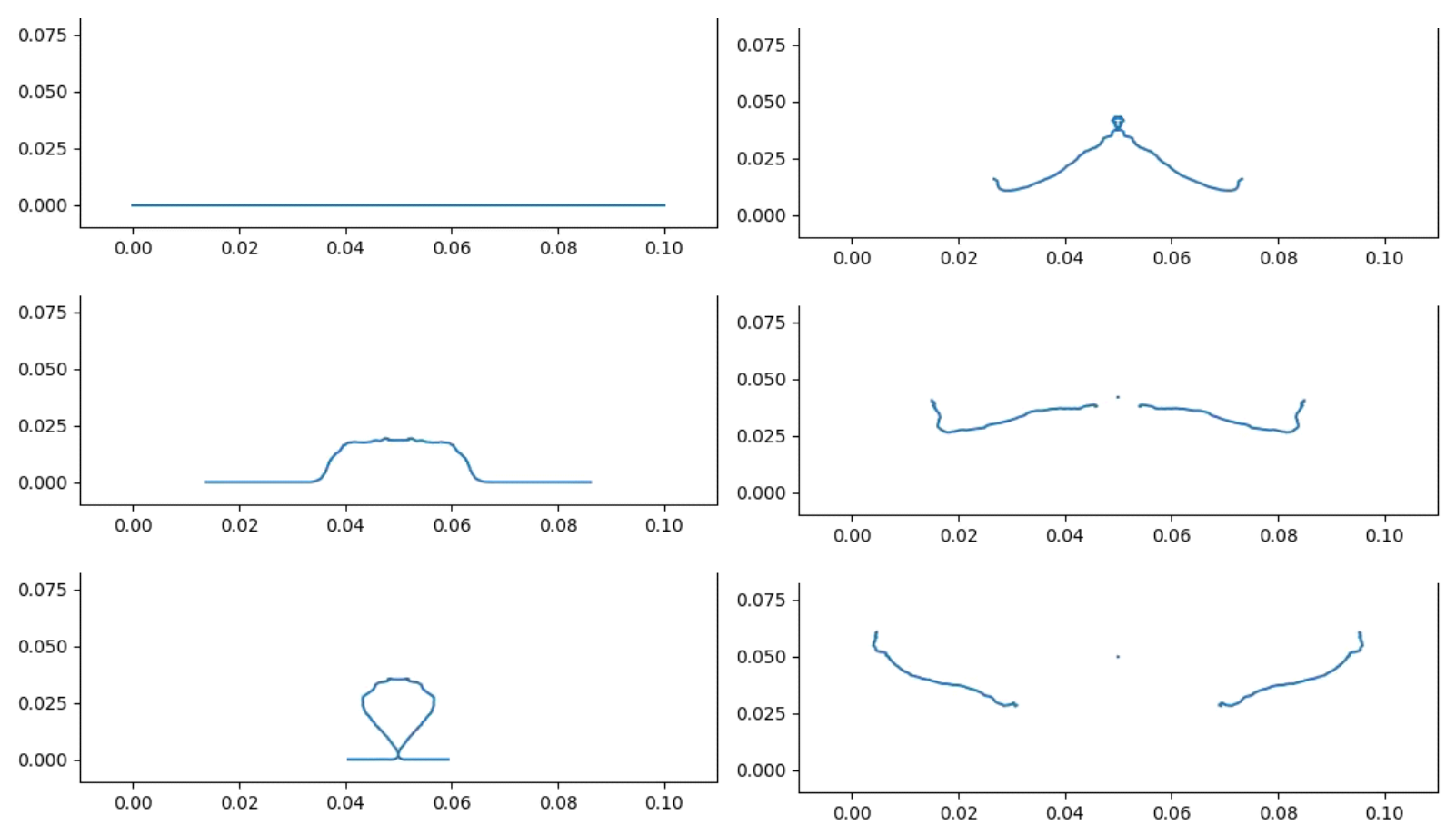

Рис.6. Развитие деформаций в нити. Внешнее давление 30 МПа. Разрыв разогнанной нити в середине. Левый столбик сверху вниз - моменты времени 0, 8, 16 мкс. Правый столбик сверху вниз - моменты времени 24, 32, 40 мкс. Координаты точек нити по осям OX и OY в метрах. 

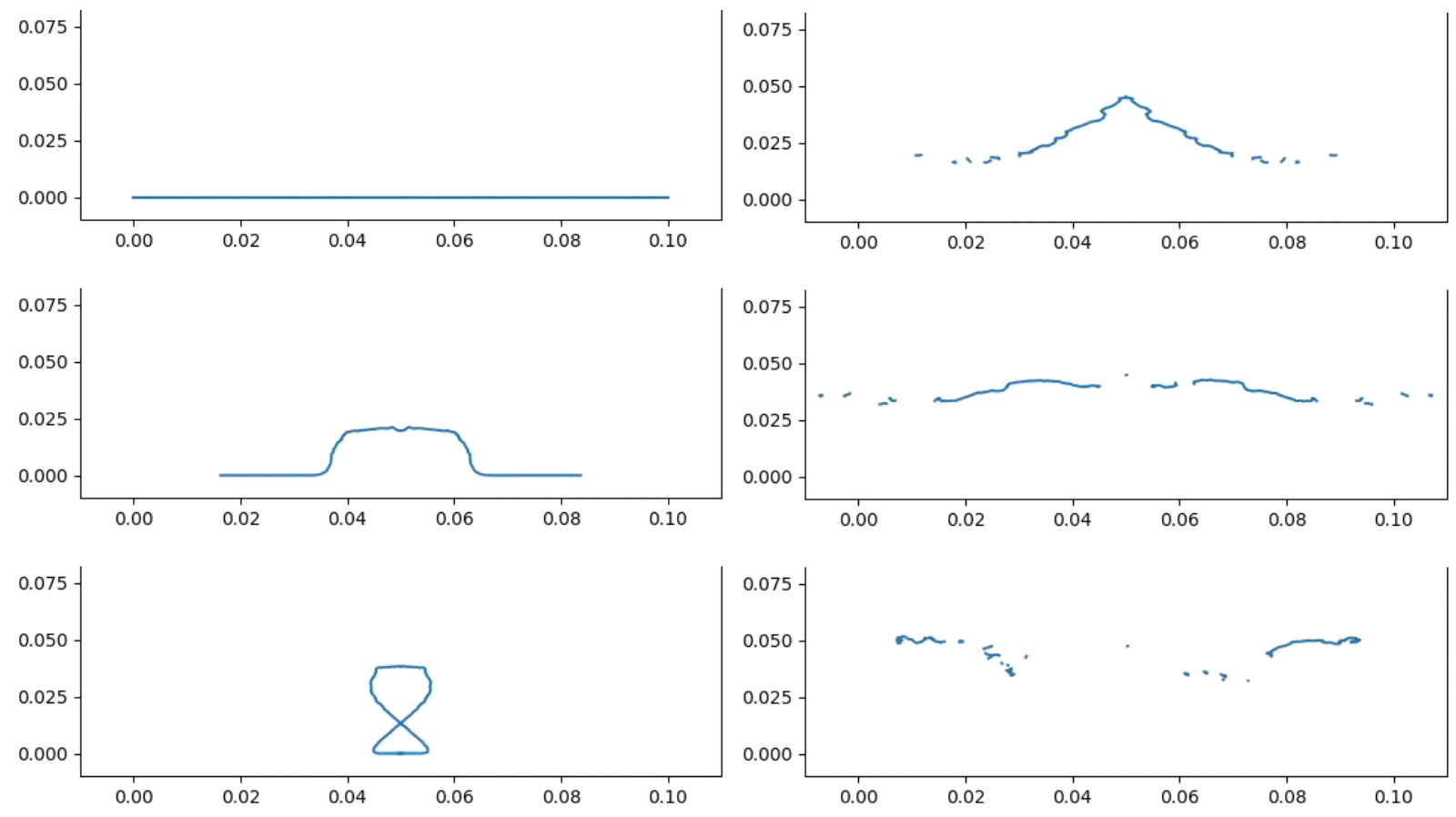

Рис.7. Развитие деформаций в нити. Внешнее давление 35 МПа. Обрыв и дробление разогнанных концов нити. Левый столбик сверху вниз - моменты времени 0, 8, 16 мкс. Правый столбик сверху вниз - моменты времени 24, 32, 40 мкс. Координаты точек нити по осям ОХ и OY в метрах.
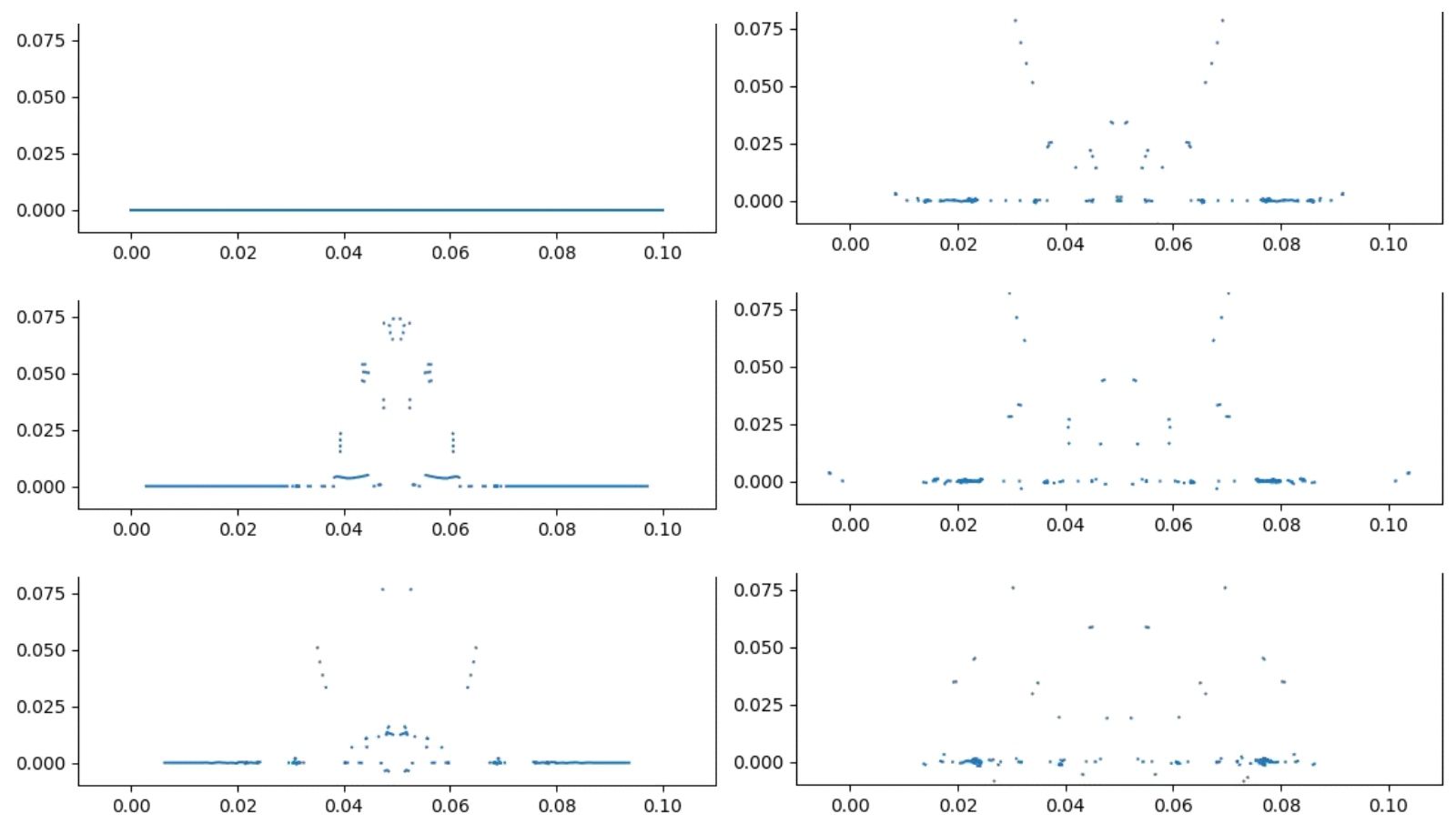

Рис.8. Развитие деформаций в нити. Внешнее давление 40 МПа. Выбивание и дробление средней части нити, затем разрушение разогнанных концевых элементов. Левый столбик сверху вниз - моменты времени 0, 8, 16 мкс. Правый столбик сверху вниз - моменты времени 24, 32, 40 мкс. Координаты точек нити по осям OX и OY в метрах. 


\section{Заключение}

Рассмотренная модель тонкой нити при действии механической ударной нагрузки позволяет выполнять расчёты высокоскоростных взаимодействий, для которых скорость движения ударника сопоставима со скоростью распространения звука в нити. Показано соответствие численных результатов аналитическому решению для режима, для которого аналитическое решение описано в литературе. Приведены расчёты различных режимов воздействия распределённой нагрузки на тонкую нить, приводящих к принципиально разной картине деформаций и разрушений нити.

Данная модель нити может использоваться для выполнения быстрых оценок поведения тканевого экрана под действием высокоскоростной нагрузки с использованием моделей, аналогичных классической модели «крест - колокол», применяемой для низкоскоростных режимов.

В рамках дальнейшей работы предполагается использовать сформулированную модель отдельной нити как основу расчётов тканевых композитов с учётом их внутренней структуры и вида плетения.

\section{СПИСОК ЛИТЕРАТУРЫ}

1. И.Ф. Кобылкин, В.В. Селиванов. Материалы и структуры легкой бронезащиты. - М.: МГТУ им. Н. Э. Баумана, 2014.

I.F. Kobylkin, V.V. Selivanov. Materialy i struktury legkoy bronezashchity. - M.: MGTU im. N.E. Baumana, 2014, $192 \mathrm{~s}$.

2. J.D. Walker. Constitutive Model for Fabrics with Explicit Static Solution and Ballistic Limit / Proc. of Eighteenth Intern. Symp. on Ballistics, 1999, San Ant., USA, p.1231-1238.

3. J.D. Walker. Ballistic Limit of Fabrics with Resin // Proc. of the Nineteenth International Symposium on Ballistics, 2001, Interlaken, Switzerland, p. 1409-1414.

4. P.K. Porval, S.L. Phoenix. Modeling System Effects in Ballistic Impact into Multi-layered Fibrous Materials for Soft Body Armor // Intern. J. of Fracture, 2005, v.135, p. 217-249.

5. Х.А. Рахматулин, Ю.А. Демьянов. Прочность при интенсивных динамических нагрузках. - М.: Логос, 2009, $512 \mathrm{c}$.

Kh.A. Rakhmatulin, Iu.A. Demianov. Prochnost pri intensivnykh dinamicheskikh nagruzkakh. - M.: Logos, 2009, $512 \mathrm{~s}$.

6. Х.А. Рахматулин, Е.И. Шемякин, Ю.А. Демьянов, А.В. Звягин. Прочность и разрушение при кратковременных нагрузках. - М.: Логос, 2008, 624 с.

Kh.A. Rakhmatulin, E.I. Shemiakin, Iu.A. Demianov, A.V. Zviagin. Prochnost i razrushenie pri kratkovremennykh nagruzkakh. - M.: Logos, 2008, $624 \mathrm{~s}$.

7. И.Б. Петров, А.И. Лобанов. Лекции по вычислительной математике. - М.: Бином, $2006,523 \mathrm{c}$.

I.B. Petrov, A.I. Lobanov. Lektsii to vychislitelnoj matematike. - M.: Binom, 2006, 523 s.

Поступила в редакцию 01.07.2019

После доработки 01.07.2019

Принята к публикации 21.10.2019 